\title{
Facteurs géographiques et sociaux de la diversité des ignames cultivées au Nord Bénin
}

\author{
Mohamed Nasser Baco ${ }^{1,4}$ \\ Gauthier Biaou ${ }^{2}$ \\ Jean-Louis Pham ${ }^{3}$ \\ Jean-Paul Lescure ${ }^{4}$ \\ ${ }^{1}$ Institut national des recherches agricoles \\ du Bénin (Inrab), \\ BP 27, \\ Parakou \\ Bénin \\ <nasser.baco@orleans.ird.fr \\ 2 Faculté des sciences agronomiques \\ du Bénin, \\ Université d'Abomey Calavi, \\ 01 BP 526 \\ Cotonou \\ Bénin \\ <gbiaou@yahoo.fr> \\ ${ }^{3}$ Institut de recherche \\ pour le développement (IRD), \\ Unité mixte de recherche (UMR) DIAPC, \\ BP 64501, \\ 34394 Montpellier cedex 05 \\ $<$ jean-louis.pham@mpl.ird.fr> \\ ${ }^{4}$ Institut de recherche pour le \\ développement (IRD), \\ Unité de recherche 168 "Dynamiques \\ environnementales entre forêt, agriculture \\ et biodiversité: des pratiques locales \\ sur la nature aux politiques publiques ". \\ Centre IRD d'Orléans, \\ 5 , rue du carbone, \\ 45072 Orléans cedex 2 \\ <nasser.baco@orleans.ird.fr> \\ <jean-paul.lescure@orleans.ird.fr>
}

\begin{abstract}
Résumé
Cette étude a été conduite dans 8 villages et 220 exploitations agricoles du Nord Bénin, où l'igname est une culture importante. La principale hypothèse de cette étude considère que le niveau de richesse variétale d'un village est influencé à la fois par la diversité ethnique et par l'enclavement (marché, route). L'étude a révélé une grande richesse variétale avec 182 cultivars d'igname observés. Elle a aussi montré l'importance des pratiques culturales, des évolutions sociales, historiques et culturelles dans la constitution du pool cultivé et la nécessité de combiner les dimensions sociale et géographique pour une bonne analyse de la diversité agricole. La thèse de l'influence réductrice du marché sur le niveau de diversité variétale n'est pas confirmée. Les Bariba, les Nago et les Gando, groupes ethniques les plus anciennement attachés à la culture de l'igname, demeurent encore aujourd'hui les meilleurs " véhicules " de sa diversité. La récente conversion des Peuls à l'agriculture et à la culture de l'igname ouvre de nouvelles perspectives pour la diversité de cette plante.
\end{abstract}

Mots clés : agrobiodiversité ; Bénin ; groupe ethnique ; igname ; variété.

Thèmes : productions végétales ; ressources naturelles et environnement.

\section{Abstract \\ Geographical and social factors of cultivated yam diversity in northern Benin}

This study aimed at contributing to the sustainable management of yam (Dioscorea sp.) diversity. It was carried out in northern Benin on 220 farms and in 8 villages. The main hypothesis considers that varietal diversity is influenced by ethnic diversity and the market. Results show that diversity remains high with 182 landraces observed in the fields. The study underlines the major role of cultivation practices and of socio-cultural determinants that depend on the history of each ethnic group. In relationship to this point, the Bariba, the Nago and the Gando, who have cultivated yams since remotest times, still remain the best social vectors of diversity. Characteristics of villages, such as links with the geographic and social levels, act on the level of diversity. The reduction of diversity brought about by market development is not confirmed. Facing the complexity of diversity determinant factors, the study concludes that the success of an in situ conservation policy will depend on the difficult choice of the area where it is carried out.

Key words: agrobiodiversity; Benin; ethnic groups; varieties; yams.

Subjects: natural resources and environment; vegetal productions.

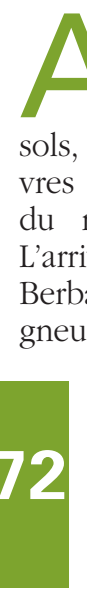

u Nord Bénin, la généralisation de la culture cotonnière a contribué à la baisse de la fertilité des sols, entraînant l'abandon des terres pauvres et le déplacement des producteurs du nord-ouest vers d'autres régions. L'arrivée continue de migrants Lokpa et Berba en provenance des zones montagneuses du nord-ouest, et d'éleveurs peuls en provenance du nord et de l'est du pays (Doevenspeck, 2004) induit des changements alimentaires. Dumont (1997) signale qu'avant les années 1970, manger du maïs était déshonorant pour le paysan Bariba du Nord Bénin censé assurer la totalité de ses besoins à partir de l'igname, voire du sorgho. De plus, l'igname rentre depuis le début des 
années 1990 dans les transactions commerciales générant de plus en plus des revenus substantiels aux paysans.

Au total, la culture de l'igname se trouve dans un environnement très changeant marqué par le développement du vivrier marchand et des migrations rurales. Notre recherche analyse les dynamiques de la diversité d'igname dans le nouveau contexte. Comment la diversité des ignames peut-elle s'enrichir à partir du développement de son marché vivrier et de la nouvelle carte ethnique induite par les migrants?

L'hypothèse principale de cette étude considère que le niveau de richesse variétale d'un village est influencé à la fois par la diversité ethnique et par l'enclavement (marché, route). De plus, la distance géographique au-delà de laquelle les échanges entre villages se maintiennent reste inconnue. La seconde hypothèse se basant sur ce constat considère que la " distance de similarité variétale " entre les villages est plus fonction de la distance ethnique entre ceux-ci que de la distance géographique.

En décrivant l'ampleur de la diversité variétale de l'igname au Nord Bénin et en tentant d'en comprendre quelques déterminants géographiques et sociaux, cette étude veut contribuer à la mise en place d'une stratégie de conservation in situ des ressources génétiques de l'igname.

\section{Matériel et méthode}

\section{Zone d'étude et choix des villages d'étude et des unités d'observation}

L'étude a été conduite au Nord Bénin, où l'agriculture constitue la principale activité. C'est une zone cosmopolite où la plupart des groupes ethniques cultivent l'igname, ce qui en fait la première région productrice.

Le Bénin compte une cinquantaine de langues (Floquet et Akker van den, 2000). Les Bariba constituent le groupe ethnique majoritaire. Dans le passé, ils s'opposaient totalement aux autres groupes par leur statut et leurs activités, orientées principalement vers la guerre, l'artisanat, accessoirement l'agriculture, mais jamais vers l'élevage. Dans la hiérarchie sociale, les Bariba formaient la caste supérieure (Lombard, 1965).

Les Peuls, à l'origine éleveurs transhumants, deviennent de plus en plus des agriculteurs-éleveurs sédentaires dans le Borgou en raison des contraintes rencontrées de nos jours lors des transhumances. Lombard (1960) les définit comme "...un groupe de statut supérieur n'ayant pas un sort enviable. Leur foi (musulmane) et leur richesse (bétail) ne leur conféraient pas un prestige aux yeux des princes Bariba qui dévaluaient tout ce qui n'était pas guerrier".

Les Gando étaient des esclaves, des captifs qui travaillaient sous le contrôle des princes Bariba et surtout des pasteurs peuls. Dans la hiérarchie ethnoprofessionnelle du Nord Bénin, les Gando se situent toujours au niveau inférieur de la société (Bierschenk, 1993).

Les Berba et les Lokpa sont des ethnies différentes, rencontrées majoritairement dans le massif de l'Atacora au nord-ouest, et jusqu'au Burkina et au Togo. Aujourd'hui, ils forment avec d'autres ethnies apparentées les principaux acteurs des dynamiques migratoires suscitées par la dégradation avancée des terres dans l'Atacora et la recherche de terres fertiles dans le Borgou, zone d'accueil. Cette situation a complexifié la configuration ethnolinguistique du Borgou.

Le groupe ethnique des Nago se rencontre à la transition entre le nord et le centre du Bénin et se trouve confronté à de fortes dynamiques migratoires.

Les 225 exploitations enquêtées, réparties dans 8 villages, ont été choisies de façon à couvrir toute la diversité des exploitations agricoles du Nord Bénin signalée dans les études diagnostiques. Les villages ont été choisis en fonction de la diversité ethnique et de leur caractère enclavé ou non par rapport aux axes routiers. Les villages situés plus au nord sont occupés majoritairement par les Bariba et les Gando, alors que les villages situés plus au sud sont occupés principalement par les Nago et les Bariba. Les Lokpa constituent l'ethnie majoritaire rencontrée dans le village de Yébessi. Le choix de ce village et des 7 autres (Agbassa, Goro, Gorobani, Kinnou kpanou, Kparo, Sèkèrè, Wari,) permet de prendre en compte les six principales ethnies productrices d'igname dans le Nord Bénin. Les villages de Yébessi et de Wari sont situés sur des routes principales de praticabilité permanente, alors que les autres villages sont situés sur des routes secondaires ou des pistes.

\section{Collecte des données}

Dans ce travail, le terme "cultivar" est entendu comme un ensemble de plantes dont les caractéristiques sont jugées suffisamment semblables par les agriculteurs pour être regroupées en une catégorie identifiée par un nom propre (Pinton et Emperaire, 2001). Chaque cultivar représente donc un "type-nommé " (Caillon et Lanouguère-Bruneau, 2005), une unité connue et manipulée en tant que telle dans la population. Jarvis et al. (2004) confirment que la représentation paysanne basée sur les noms des cultivars constitue un point d'entrée important pour l'étude de la diversité variétale.

La constitution d'une base de données sur les exploitations observées et les cultivars recensés a permis de comparer la diversité entre les villages et entre les ethnies. Nous avons eu recours à des données qualitatives complémentaires pour analyser les déterminants sociaux et géographiques et leurs impacts sur la diversité des ignames cultivées.

\section{Outils d'analyse}

Les pools variétaux ont été analysés à l'aide de différents outils. Nous avons utilisé les statistiques développées récemment par Colwell et al (2004), en utilisant le logiciel libre Estimates.

Les courbes d'accumulation représentent le nombre cumulé de cultivars rencontrés en fonction du nombre d'informateurs enquêtés. Nous utiliserons ici la courbe d'accumulation de Mao Tau qui, à partir des données observées, s'affranchit du biais introduit par l'ordre des enquêtes. Nous utilisons comme indice d'estimation de la richesse variétale au niveau de la population, celui de Chao2 (moyenne, et écart type) proposé par Chao et al (2005), basé sur les abondances. Ces deux indices ont été calculés en réalisant 100 retirages aléatoires de l'ordre des enquêtes. Enfin, les similarités entre villages, ou entre groupes ethniques, ont été évaluées par l'indice de Chao-Sorensen qui, se basant sur les abondances (ici le nombre de buttes cultivées par cultivar), utilise plus d'information que l'indice classique de Sorensen basé sur les simples fréquences.

\section{Résultats et discussion}

\section{Facteurs géographiques de la diversité d'igname}

Répartition par village

Le tableau 1 permet de comparer les richesses variétales cultivées par les villa- 
Tableau 1. Nombre de cultivars observés par village.

Table 1. Observed cultivars within studied villages.

\begin{tabular}{llcccccccc}
\hline Paramètres & $\begin{array}{l}\text { Intervalle } \\
\text { de confiance à 95 \% }\end{array}$ & Agbassa & Goro & Gorobani & $\begin{array}{l}\text { Kinnou } \\
\text { Kpanou }\end{array}$ & Kparo & Sèkèrè & Wari & Yebessi \\
\hline Diversité observée & Valeur minimum & 27 & 32 & 40 & 29 & 50 & 43 & 28 & 31 \\
& Moyenne & 33 & 39 & 46 & 35 & $\mathbf{5 4}$ & $\mathbf{5 2}$ & 31 & 33 \\
& Valeur maximum & 38 & 45 & 52 & 41 & 59 & 61 & 40 & 43 \\
\hline
\end{tabular}

ges. Les villages ayant le même nombre de cultivars sont ceux dont les intervalles de confiance se recoupent. En revanche, ceux dont les intervalles ne se chevauchent pas ont des différences de pools variétaux significatives au seuil de $5 \%$. Les villages de Gorobani, Sèkèrè, et Kparo gèrent les plus grands pools (environ 50 cultivars). Ils sont suivis de Goro et Kinnou kpanou (aux alentours de 40). Avec une trentaine de cultivars, les villages de Wari, Yebessi et Agbassa, possèdent les diversités les plus faibles.

Les indices de similarité (Chao-Sorensen) entre les villages (figure 1) montrent que le village de Yébessi se distingue des autres avec des valeurs toujours inférieures à 0,25 . Ce sont les couples de villages Goro-Kinnou Kpanou, Gorobani-Kparo, Agbassa-Goro, Agbassa-Kinnou-kpanou, Gorobani-Sèkèrè, Gorobani-Wari, KparoSèkèrè, Kparo-Wari, SèkèrèWari (ayant des coefficients proches de 1) qui partagent le plus de cultivars.

En cherchant à comprendre si la distance métrique qui sépare les villages constitue un facteur explicatif de la similarité ou non de leur pool, il ressort - tableau 2 - que les couples de villages ayant une grande similarité variétale (indice $>80$ ) sont distants en moyenne de $33 \mathrm{~km}$. Au-delà de $48 \mathrm{~km}$, la similarité se réduit. Les villages les moins similaires sont séparés d'une distance moyenne de $193 \mathrm{~km}$.

Le test de comparaison (test t de Student) de moyennes de distance au sein des villages à grande similarité variétale et au sein des villages à faible similarité variétale est hautement significatif. Il existe alors une relation entre la distance des villages et leur similarité variétale.

Le critère "enclavement" repose sur l'accessibilité des villages et la présence de marché. Les villages enclavés sont donc des terroirs qui sont mal intégrés aux mécanismes du marché. En combinant ce critère avec celui de la diversité ethnique, les diversités estimées (Chao2) des groupes de villages ainsi obtenus sont fournies dans le tableau 3.

Les villages les moins enclavés et les plus enclavés ont respectivement des moyen- nes estimées de 65 et 71 cultivars. La comparaison de ces moyennes n'est pas significative $(p=0,56)$; il n'existe pas de relations entre le nombre de cultivars d'un village et son niveau d'enclavement. Les villages les plus ethniquement divers et ceux qui le sont le moins possèdent respectivement des moyennes de 74 et 56 cultivars. La comparaison des moyennes de cultivars de ces deux classes est significative. Le niveau de diversité variétale d'igname d'un village est lié à sa diversité ethnique.

\section{Déterminants géographiques de la gestion de la diversité agricole}

L'enclavement est une " absence d'accès au marché dans un espace donné" (George et Verger, 1996). Cette définition s'appuie sur l'idée de "marché " et suppose que toute situation territoriale (par exemple la diversité d'un village) prend sa signification dans l'échange, la relation, la complémentarité. L'existence de voies d'accès convenables permet aux villages d'échanger avec l'extérieur, d'organiser des marchés, et donc d'orienter la production en fonction d'une demande extérieure. Dans ces conditions, selon certains auteurs (Bellon 2001) la diversité cultivée tend à s'uniformiser voire à se réduire.

Les villages positionnés sur les grands axes ont développé ces dix dernières années un marché autour de l'igname et en exportent de plus en plus au-delà des frontières (Nigeria, Niger, Burkina). Cela résulte de la politique de "villagisation" du premier président du Bénin (Hubert Maga) qui, à la faveur de l'indépendance du pays en 1960, a opté pour le déplacement vers les grands axes des communautés vivant en retrait dans les zones non accessibles. Cette mesure politique a entraîné entre 1960-1963, la création de nouveaux villages tels que Yébessi et Wari, qui bénéficient aujourd'hui de leur position géographique pour développer des marchés locaux.

Malgré ce développement marchand, les villages ouverts sur l'extérieur (moins enclavés) ont des pools variétaux qui ne se différencient pas significativement des villages plus enclavés. La thèse de l'influence réductrice du marché sur le niveau de diversité variétale ne semble donc pas confirmée en termes de gamme variétale; en revanche, plusieurs études sur l'agrobiodiversité de l'igname dans la région ont montré qu'un petit nombre de variétés représentent l'essentiel des surfaces cultivées (Baco et al., 2004 ; Vernier et Dansi, 2006). Ainsi, Vernier et Dossou (2002) ont montré que dans la même région 2 cultivars chez les Dioscorea rotundata précoces couvrent plus de $50 \%$ des surfaces cultivées en igname et 5 plus de $80 \%$ (respectivement 4 et 9 chez les cultivars tardifs).

Les résultats montrent aussi qu'il existe des villages fortement et faiblement similaires au point de vue variétal. Cette présence de similarité entre les villages traduit l'existence d'échanges variétaux entre ces derniers.

Ainsi, les flux migratoires peuvent également avoir un impact sur la diversité. Jusqu'en 1985, les producteurs du NordOuest ont abandonné leurs terres dégradées pour rejoindre les autres régions ou le Nigeria à la recherche de terres fertiles ou d'opportunités meilleures. À cette date, le Nigeria a expulsé les étrangers et s'est enfoncé dans une crise économique qui le rend moins attractif pour les migrants. En revanche, les flux intra- et interrégionaux à l'intérieur du Bénin se poursuivent à un rythme soutenu (Bruntrup, 1997). Les producteurs dans leur déplacement à la recherche de nouvelles terres emportent, introduisent et adoptent de nouveaux cultivars rendant ainsi dynamique la gestion de la diversité dans des terroirs d'accueil.

\section{Facteurs sociaux de la diversité}

Répartition des cultivars

entre les groupes ethniques

Les courbes d'accumulation de Mao Tau calculées pour chaque ethnie se différencient très rapidement (figure 2). Une trentaine d'informateurs par ethnie 


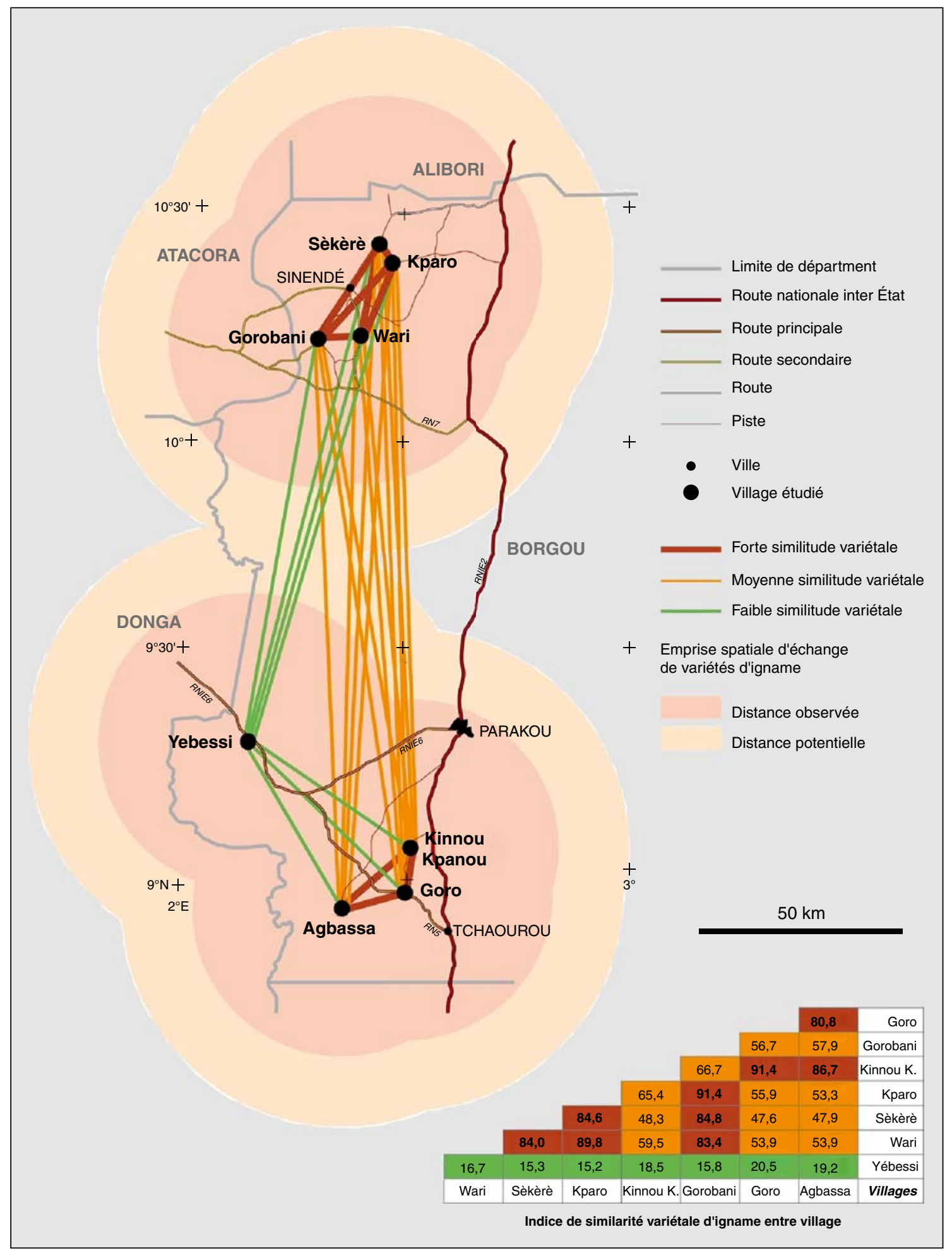

Figure 1. Distance de similarité variétale entre villages et importance des ethnies par village.

Figure 1. Varietal similarity distance between villages and ethnic importance by village.

suffisent amplement pour mettre en évidence les différences entre les ethnies, et trois groupes semblent se différencier.
Les Bariba détiennent le pool de cultivars le plus important. Ils sont suivis par les Nago, Gando. Les Lokpa, Peuls et Berba présentent une richesse en cultivars similaire, la plus faible parmi les groupes ethniques étudiés. 
Tableau 2. Comparaison des distances moyennes entre villages similaires et les villages moyennement et faiblement similaires sur le plan variétal distingués à partir des coefficients de similarité (Chao-Sorensen).

Table 2. Mean distance comparison between villages of similar and lower similar varietals, classified through similarity index (Chao-Sorensen).

\begin{tabular}{|c|c|c|c|c|c|c|}
\hline Similarité variétale & $\begin{array}{c}\text { Nombre } \\
\text { de couples } \\
\text { de village }\end{array}$ & $\begin{array}{c}\text { Distance } \\
\text { moyenne } \\
\text { entre villages } \\
(\mathrm{km})\end{array}$ & $\begin{array}{l}\text { Écart } \\
\text { type }\end{array}$ & $\begin{array}{l}\text { Erreur standard } \\
\text { moyenne }\end{array}$ & $\begin{array}{c}\text { IC } 95 \% \\
\text { limite } \\
\text { inférieure } \\
\text { (km) }\end{array}$ & $\begin{array}{c}\text { IC } 95 \% \\
\text { limite } \\
\text { supérieure } \\
\text { (km) }\end{array}$ \\
\hline Villages à grande similarité & 9 & 32,89 & 23,20 & 7,73 & 7,74 & 48,35 \\
\hline Villages à similarité faible & 19 & 193,32 & 48,95 & 11,23 & 170,90 & 215,78 \\
\hline \multicolumn{7}{|c|}{$t=9,281 ; d d l=26 ; p=0,000$} \\
\hline
\end{tabular}

$t$ : valeur du test ; ddl : degré de liberté ; $p$ : probabilité ; IC : intervalle de confiance.

\section{Tableau 3. Diversité variétale moyenne estimée en fonction des groupes de villages obtenus par combinaison des critères d'enclavement et d'ethnie.}

Table 3. Comparison of estimated varietal diversity by considering ethnic and market criteria.

\begin{tabular}{|c|c|c|c|c|c|c|c|}
\hline & & \multicolumn{4}{|c|}{ Enclavement } & \multirow{3}{*}{\multicolumn{2}{|c|}{ Moyenne }} \\
\hline & & \multicolumn{2}{|c|}{ Moins } & \multicolumn{2}{|c|}{ Plus } & & \\
\hline & & Villages & Cultivars & Villages & Cultivars & & \\
\hline \multirow{7}{*}{$\begin{array}{l}\text { Diversité } \\
\text { ethnique }\end{array}$} & Moins & Wari & 40 & Kparo & 68 & 56 & $t=2,35$ \\
\hline & & Yekessi & 59 & & & & $p=0,05$ \\
\hline & Plus & Goro & 69 & Agbassa & 69 & 74 & \\
\hline & & Kinnou & 68 & Gorobani & 77 & & \\
\hline & & Sèkèrè & 88 & & & & \\
\hline & Moyenne & & 65 & & 71 & & \\
\hline & & \multicolumn{3}{|c|}{$t=0,62$ et $p=0,56$} & & & \\
\hline
\end{tabular}

$t$ : valeur du test ; $\mathrm{p}$ : probabilité.

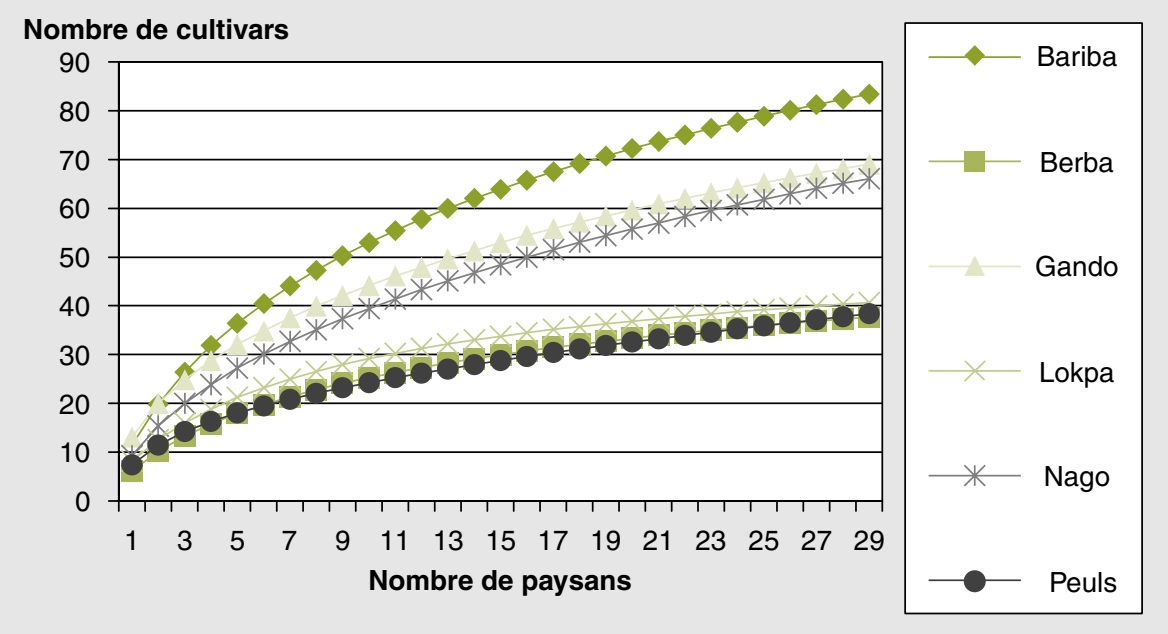

Figure 2. Courbe d'accumulation de la richesse spécifique d'igname détenue par les ethnies en fonction de la taille de l'échantillon.

Figure 2. Accumulation curve of the specific richness of yams held by the ethnic groups.
La similarité du pool de cultivars entre les différents groupes ethniques (tableau 4) montre que les Bariba partagent une grande partie de leur pool avec toutes les ethnies, sauf avec les Lokpa. Ces derniers apparaissent comme un groupe particulièrement original, leur indice de similarité avec les autres groupes restant toujours inférieur à 0,25 . Une grande similarité entre les pools de cultivars détenus par les groupes Berba et Peuls est observée.

Déterminants ethniques de la gestion de la diversité agricole

La forte similarité en cultivars observée entre Bariba et Nago peut s'expliquer par le lointain substrat social qu'ils ont en commun. Les Nago et les Bariba sont tous deux originaires du Nigeria qu'ils ont quitté pour créer des royaumes monarchiques, de type féodal, organisés selon des relations complexes de vassalité dans le Moyen Bénin et le Nord Bénin (Lombard, 1965). Après l'abolition de l'esclavage par le pourvoir colonial en 1896, ces deux royaumes esclavagistes ont été fragilisés (Alber, 1997). Le rapport de méfiance qu'ils avaient l'un pour l'autre alla en s'estompant et désormais les membres de ces deux groupes développent et entretiennent des relations amicales où chacun revendique aujourd'hui encore d'avoir constitué par le passé la caste hiérarchique la plus importante.

Par ailleurs, les Bariba et les Gando possèdent une diversité en cultivars d'igname plus importante que les autres ethnies du fait de leur histoire commune au cours de laquelle l'igname a toujours été valorisée. Les Gando travaillaient sous le contrôle des princes Bariba. La plupart d'entre eux étaient des prisonniers de guerre; quelques-uns étaient des Bariba qui avaient été abandonnés dans leur enfance à la suite d'une naissance jugée "maléfique" ou d'une dentition "anor- 


\section{Tableau 4. Valeur des coefficients de similariété variétale (Chao-Sorensen) par couple d'ethnies.}

Table 4. Similarity index within the ethnic groups (Chao-Sorensen).

\begin{tabular}{lccccc}
\hline Ethnies & Bariba & Berba & Gando & Lokpa & Nago \\
\hline Berba & 0,81 & & & & \\
Gando & 0,78 & 0,60 & & & \\
Lokpa & 0,23 & 0,19 & 0,11 & & \\
Nago & 0,85 & 0,77 & 0,56 & 0,24 & \\
Peuls & 0,86 & 0,84 & 0,63 & 0,12 & 0,86 \\
\hline
\end{tabular}

male " (Lombard, 1960). La similarité des cultivars rencontrés chez les Bariba et les Gando peut être reliée à l'assimilation de la culture du maître analysée par Meillassoux (1989).

Cette relation séculaire d'esclavage n'a pas existé entre les Nago et les Gando, ce qui expliquerait la faible similarité en cultivars entre ces deux groupes, bien que chacun d'eux soit lié aux Bariba.

Les groupes ethniques Lokpa, et Berba détiennent moins de cultivars que les autres du fait probablement de leur statut commun de "migrants". Contrairement aux Lokpa, les Berba ont une similarité plus forte avec les autres ethnies. Les Lokpa ont quitté leurs villages des zones montagneuses de l'Atakora en emportant leurs cultivars il y a une quarantaine d'années à la suite d'une mesure politique, alors que les Berba quittent de nos jours leurs aires géographiques (nordouest) pour le Borgou du fait de la pauvreté de leurs sols et pour servir de maind'œuvre agricole. Dans leur migration, ils introduisent rarement leurs cultivars et se contentent en général des cultivars trouvés dans les terroirs d'accueil.

\section{Conclusion}

La remarquable diversité de cultivars d'ignames observée dans le Nord résulte de pratiques culturales autant que de faits sociaux, économiques et culturels. Malgré les conditions environnementales similaires, les ethnies étudiées cultivent différents cultivars. Les Lokpa et les Berba préfèrent les gros tubercules, les Bariba et les Gando optent pour les cultivars de taille moyenne, d'autres, en revanche, préfèrent les cultivars à petits tubercules. Les groupes ethniques gèrent différemment la diversité qu'ils détiennent. La ressem- blance du matériel biologique cultivé par des communautés différentes tient à leur histoire commune depuis des décennies. Les Bariba et les Gando, groupes ethniques les plus anciennement attachés à la culture d'igname, demeurent encore aujourd'hui les meilleurs "véhicules sociaux " de sa diversité. Le début de conversion des Peuls à l'agriculture ouvre de nouvelles perspectives pour cette diversité.

L'hypothèse qui considère que le marché contribue à réduire la diversité agricole est nuancée. L'intégration des villages dans les réseaux marchands favorise des échanges avec l'extérieur et conduit dans le temps à une similarité entre villages appartenant aux mêmes réseaux d'échanges.

L'étude indique clairement le lien entre la diversité ethnique et la diversité variétale et la prédominance des facteurs culturels (ethniques) sur les facteurs géographiques (enclavement) dans la structuration du pool variétal des villages. La richesse spécifique gérée sur une exploitation agricole est plus fonction de son appartenance ethnique que de son espace géographique. Cependant, la combinaison des facteurs géographiques et ethniques est essentielle pour une dynamique variétale au profit des paysans. La photographie géographique et ethnique indique aussi la nécessité d'une prise en compte multiscalaire régionale et locale pour sauvegarder les processus et interactions socio-économiques en faveur de la diversité des ignames.

\section{Références}

Alber E. Le pouvoir local face aux mutations au niveau de l'État. Le cas d'un village Bariba. Cah Etud Afr 1997 ; 145 : 137-56.

Baco MN, Tostain S, Mongbo RL, Dainou O, Agbangla C. Gestion dynamique de la diversité variétale des ignames cultivées (Dioscorea cayenensis- $D$. rotundata) dans la commune de Sinendé au nord Bénin. PGRNewletters $2004 ; 139$ : 18-24.
Bellon MR. Demand and supply of crop intraspecific diversity on farms : towards a policy framework for on-farm conservation. Mexico : International Maize and Wheat Improvement Centre (CIMMYT), 2001.

Bierschenk T. The creation of a tradition: Fulani chiefs in Dahomey-Benin from the late 19 th century. Paideuha $1993 ; 39$ : 217-44.

Bruntrup M. Agricultural price policy an dits impact on production, income, employment and the adoption of innovations. A farming base analysis of cotton in northern Benin. Frankfurt : Peter, 1997.

Caillon S, Lanouguère-Bruneau V. Gestion de I'agrobiodiversité dans un village de Vanua Lava (Vanuatu): stratégies de sélection et enjeux sociaux. J Soc Océanistes 2005; 1 : 120-1.

Chao A, Chazdon RL, Colwell RK, Shen TJ. A new statistical approach for assessing compositional similarity based on incidence and abundance data. Ecol Lett $2005 ; 8$ : 148-59.

Colwell RK, Mao CX, Chang J. Interpolating, extrapolating, and comparing incidencebased species accumulation curves. Ecology based species accum
$2004: 85: 2717-27$.

Doevenspeck M. Migrations rurales, accès au foncier et rapport interethniques au sud du Borgou (Bénin). Une approche méthodologique plurielle. Afr Spectr 2004 ; 39 : 359-80.

Dumont R. Étude morpho-botanique des ignames Dioscorea rotundata et Dioscorea cayenensis cultivées au Nord-Bénin. Agron Trop $1997 ; 32: 225-41$.

Floquet A, Akker van den E. Ethnic group and settlement patterns in Bénin. In : Graef $F$, Lawrence $\mathrm{P}$, von Oppen M, eds. Adapted Farming in West Africa: Issues, potentials and ming in West Africa: Issues, potentials and
perspectives. Stuttgart: Verlang Ulrich E. Grauer, 2000.

George P, Verger F. Dictionnaire de la Géographie. Paris : PUF, 1996.

Jarvis DI, Zoes V, Nares D, Hodgkin T. On-farm management of crop genetic diversity and the Convention on Biological Diversity programme of work on agricultural biodiversity. Plant Gen Res Newslett 2004 ; 138 : 5-17.

Lombard J. La vie politique dans une ancienne société de type féodal: Les Bariba du Dahomey. Cah Etud Afr $1960 ; 3: 5-45$.

Lombard J. Structures de type "féodal " en Afrique noire. Études des dynamiques et des relations sociales chez les Bariba du Dahomey. Paris : Mouton, 1965.

Meillassoux C. "Échange". Encyclopédie Universalis, éd, 1989

Pinton F, Emperaire L. Le manioc en Amazonie brésilienne: diversité variétale et marché. Genet Sel Evol 2001 ; 33 : 491-512.

Vernier P, Dansi A. Participatory assessment of local yam cultivars (D. cayenensis and $D$. rotundata) in Benin. PGRNewletters 2006. $147: 38-46$.

Vernier P, Dossou RA. Adaptation of yam (Dioscorea spp) cultivation to changing environment and economic constraints in Benin, STRC, "Potential of root crops for food and industrial resources", Tsukuba, Japan, 10-16/09/2000, ISTRC, 2002. 